\title{
PALPABLE CONTRACTILE PYLORIC TUMOURS IN THE NEWLY BORN
}

\author{
BY \\ W. S. CRAIG \\ From the Department of Paediatrics and Child Health, the University of Leeds
}

(RECEIVED FOR PUBLICATION AUGUST 19, 1955)

\begin{abstract}
A palpable tumour which contracts and relaxes is generally accepted as conclusive confirmatory evidence of symptoms and signs suggestive of hypertrophic pyloric stenosis in infancy. The writer's experience, in common with that of other workers, is that a contractile tumour is present in $98 \%$ of infants with pyloric stenosis examined for the first time over the age of 2 weeks. A detailed study carried out over the past two and a half years suggests that the same significance cannot invariably be attached to the palpation of a contractile pyloric tumour in babies under 2 weeks of age. The purpose of this article is to describe the findings which have given rise to these doubts.

The term 'tumour' is used throughout as meaning a clinically detectable swelling.
\end{abstract}

\section{Origin of the Study}

The study began as a result of the unexpected finding of a contractile pyloric tumour in a baby girl aged 7 days, reported on account of a tendency to recurrent regurgitation and unsatisfactory weight progress from the third day of life. There were no obvious factors arising in the mother or infant to account for the baby's symptoms which were disconcerting rather than alarming. Regurgitation gradually lessened and eventually ceased with regular daily stomach lavages and without any modification of the feeding routine. A large quantity of mucus was present in the early lavages. Discharged from hospital on the fourteenth day of life, the infant was examined at intervals for a further period of two months. The tumour became less readily palpable at successive examinations, and was no longer detectable two months after the baby had been discharged from hospital. Progress during these two months was symptomless and uneventful.

By what can only be described as sheer coincidence a pyloric tumour was palpated in similar circumstances in another baby within a few weeks of the one described.

\section{Choice of Clinical Material}

In view of the above accidental findings it was decided to carry out organized clinical studies on two series of cases, viz.:

A. Infants of less than 14 days of age in whom an unsatisfactory weight progress was associated with pronounced posseting, regurgitation or/and vomiting over a period of more than 48 hours. (Babies with symptoms which might have been contributed to by nasal catarrh, swallowing air, abnormalities of the palate or mandibular joint, or by difficulties related to the mother's nipples and breasts were excluded. No baby with fever, detectable infection or enteritis was included. Constipation was not a feature in any case.)

Twenty-one babies are included in this series. Of them, 18 were born in Leeds Maternity Hospital. Two were admitted to the hospital from their homes and one from a nursing home. Premature babies are not included.

B. A control series consisted of 115 healthy newborn infants who showed uninterrupted progress, and who did not experience any feeding difficulties. All the infants were born at term in the Leeds Maternity Hospital. Their ages at the time of examination ranged from 5 to 7 days. In other respects the babies were unselected, no account being taken of sex, place in family or the method of feeding.

\section{Method of Examination in Hospital}

The same procedure was adopted for all infants in series A and B. After lavage of the baby's stomach, abdominal examination was carried out while the baby was being fed. Palpation was begun immediately before the start of a feed, interrupted occasionally to allow the infant to 'break wind', and continued for some little time after completion of the feed. Feeding during the time of examination was invariably by bottle. This procedure was adopted to facilitate examination. The milk given was human or cow's according to that which the baby had been receiving as a routine in the lying-in 
ward. The results of gastric lavage were noted in every case. All babies were examined and the results recorded independently by a second competent observer.

\section{Methods of Follow-up}

As indicated below, contractile pyloric tumours were palpated in the majority of infants included in the first series $A$, and in a proportion of those included in the control series $B$. Babies in whom a tumour was palpated were re-examined by the writer at intervals extending over a number of weeks or months until such time as the tumour could no longer be felt. In most instances the infant was brought to hospital for re-examination, in which event the procedure followed was similar to that already described. Infants living at a considerable distance from the hospital were visited personally in their homes and examined at the baby's customary feeding time, but preliminary gastric lavage was not practicable.

Infants in whom no tumour was palpated during the time they were in the Maternity Hospital were not followed up personally. They were, however, the subject of follow-up visits by health visitors in the employ of the City of Leeds Maternity and Child Welfare Department.

\section{Observations concerning the Presence or Absence of a Tumour}

Examination of the abdomen after gastric lavage facilitates palpation of individual intra-abdominal structures. It is possible to palpate the pylorus in a large number of newborn infants. Palpation throughout the time of a feed facilitates detection of changes in the pylorus which accompany the gradual filling of the stomach.

In a small number of infants the pylorus could not be felt at any time-before, during or after a feed. In others it was recognizable as a short ribbon-like or worm-like structure subject to changes in firmness or tone. Not infrequently a vague sense of soft thickening was felt at intervals at a point in the length of the pylorus. Less frequently but not rarely, the local thickening was characterized by greater firmness and by a fusiform or spheroidal shape. Pyloric thickening in its most unmistakable form was characterized by a combination of precise definition and pronounced firmness. Exceptionally the firmness assumed a degree of extreme hardness.

The shape of the thickening varied. In some it was round or spheroidal; in others it resembled that of a spinning top. 'Hardening' only became detectable after the start of feeding. Evidence of defined shape and contraction usually appeared after approximately $1 \mathrm{oz}$. of feed had been taken. Local thickening and hardening, when present, were not persistent. They tended to appear and disappear.

For the purpose of the study a tumour has been regarded as being present if the palpable pyloric thickening (a) had definition, whether spherical or 'spinning top' in shape, (b) had an appreciably firm or distinctly hard consistence, $(c)$ was felt to contract and relax repeatedly, $(d)$ was felt on each occasion at the time of successive examinations and $(e)$ was felt by more than one competent observer.

Infants in whom all these five conditions were not satisfied were not included among those with tumours. This involved the exclusion of all borderline cases.

Importance was not attached to the apparent size of the tumour. The fact that definition, consistence and contraction were detectable was accepted as having greater significance than size, more especially as in older infants with pyloric stenosis clinical impressions concerning the size of the tumour are frequently not borne out at operation.

\section{Clinical Findings}

A: Selected Series (21 Infants). Of the 21 infants constituting this series, 11 were males and 10 females. There were 11 first-born babies. The place in the family of the remainder was as follows: second, seven babies; fourth, one; fifth, one; and eleventh, one baby.

A tumour was palpated in 17 of the series. An indefinite localized thickening was felt in two, and the findings were completely negative on examination of two other babies. Peristalsis was seen in all infants in whom a tumour was felt, and in two of those in whom no tumour was detected. A feature of all 21 infants was the large amount of viscid mucus in the gastric lavage.

Three male infants (two first-born and one a second child) were referred to a surgical colleague. In each case operation resulted in the immediate relief of symptoms, the severity and persistence of which had indicated the need for surgical intervention (Fig. 1). Management of the other 18

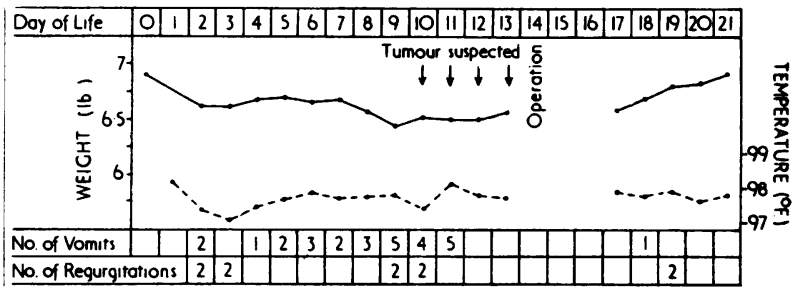

Fig. 1.-Chart of Baby 3, Series A (see Appendix). 
infants was limited to gastric lavage. Symptoms subsided within seven to 14 days of onset. All 21 infants were followed up for a period of not less than six months. In no instance was there a return of symptoms. The tumours which were felt in 14 infants, but which were not treated surgically, continued to be palpable after discharge from hospital for a period of time varying from four weeks to three months (Fig. 2).

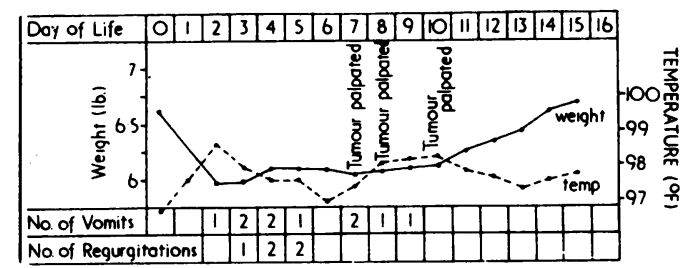

Fig. 2.-Chart of Baby 7, Series A.

Fourth child, female, normal delivery following medical induction for maternal pre-eclampsia. Hard spheroidal contractile tumour of moderate size palpable for 10 weeks. Bottle-fed on pooled human milk for five days, thereafter on dried cow's milk. No operation.

B: Control Series (115 Infants). Infants included in this series were all born in hospital and were under clinical supervision from the time of birth. There were 69 first-born babies, of whom 39 were males and 30 females. Of the 46 infants who were not first-born, 29 were males and 17 females.

In the total control series of 115 babies a tumour was felt in 16 males (including 13 first-born) and nine females (including five first-born). Mucus in large amounts was a feature of the gastric lavage in 19 of these 25 babies. Of the infants in whom a tumour was felt, 21 were followed up for a period of not less than six months. In 10 of them a tumour was no longer palpable six weeks after discharge from hospital and in the remaining 11 the tumour had disappeared by the age of 3 months.

No tumour was palpable in 90 babies in the series. Large amounts of mucus were a feature of the gastric lavage in only five of these infants. Trace was lost of three babies. One infant (a male and

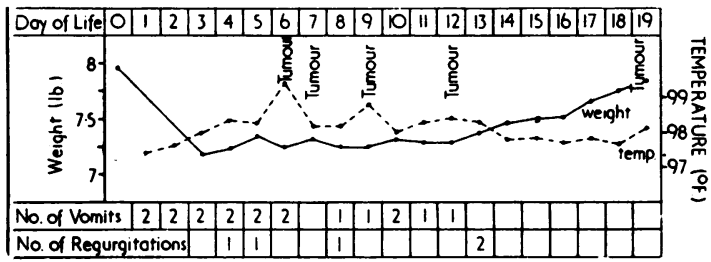

Fig. 3.-Baby 15, Series A.

Second child, female, prolonged labour, instrumental delivery, no clinical signs of intracranial irritation. Firm, fusiform contractile tumour palpable until the age of 4 months. Breast fed throughout, no operation. a first child) developed symptoms of pyloric stenosis at the age of 4 weeks and was operated upon at the age of $4 \frac{1}{2}$ weeks. Not one of the remaining 86 babies had symptoms attributable to primary gastro-intestinal dysfunction in the first six months of life.

Peristalsis of varying degree was seen in 15 infants in whom a tumour was detected and in 40 in whom no tumour was felt. Peristalsis was most pronounced in babies whose gastric lavage contained notably large amounts of mucus.

One of the infants with no tumour in the control series (B), was the twin sister of a male (eleventh) child who was included in the first series (A) and in whom a tumour was readily palpable.

\section{Supplementary Studies}

X-ray Screening (62 Infants). Sixty-two babies were screened after being given a barium feed. Screening was carried out as a routine at 3 p.m., the infant's last previous feed having been given at 9 a.m. on the same day. The 62 babies consisted of 16 (including the three who were operated on) in Series A and 46 in Series B. At the time of screening the radiologist had no knowledge of the clinical findings in the infants referred to him. Included in the 62 babies were 25 (12 from Series $A$ and 13 from Series B) in whom a contractile

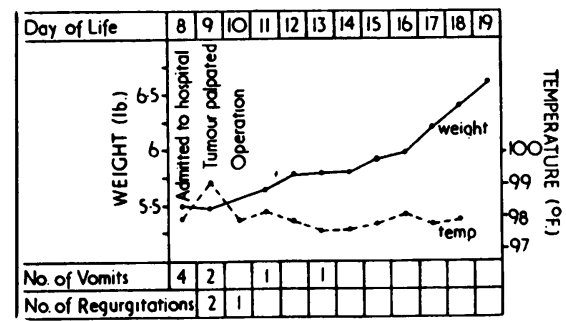

FIG. 4.-Baby 18, Series A (see Appendix).

First child, male, uneventful labour and delivery, birth weight $7 \mathrm{lb}$. Notably large, hard, contractile tumour palpated; gross gastric distension and marked peristalsis. No tumour found at operation. Cessation of symptoms after Rammstedt incision.

pyloric tumour had been palpated; 29 (all from Series B) in whom no tumour had been felt; and eight (one from Series A and seven from Series B) in whom tumours had been felt, but which because they did not satisfy the conditions enumerated on page $\mathbf{4 8 5}$ were excluded as border-line cases.

The reported findings on screening can best be summarized as follows:

In INFANTS (29) IN WHOM No Tumour WAS Felt. In 23, nothing abnormal was noted; in four, there was initial lack or slowness of peristaltic activity for 
a period varying from two to 10 minutes; and in two infants, rapid emptying in association with vigorous peristalsis, was a feature.

IN INFANTS (8) IN WHOM a 'BORDER-LINE' TUMOUR WAS FeLt. In five, immediate vigorous peristalsis and rapid emptying of the stomach were noted; in two, initial peristalsis was slow to appear and emptying of the stomach did not begin for a few minutes; and in one infant, peristaltic activity and emptying of the stomach were normal.

IN INFANTS (25) IN WHOM A CONTRACTILE TUMOUR Was Palpated. In five, nothing abnormal was noted; and in six there was initial delay in peristaltic activity and emptying of the stomach (with slight lengthening of the pyloric canal in two) and considerable delay in one. In 11 other infants, there were noted immediate markedly vigorous peristaltic contractions, notably rapid emptying of the stomach and wide dilatation of the pyloric canalthe emptying in two instances being reported on as 'amazingly rapid'.

The three remaining cases in this group consisted of those submitted to operation. In the two in whom a tumour was subsequently confirmed at operation (Appendix: Baby 3, Series A and Baby 9, Series A) screening showed gross delay in emptying of the stomach with associated distension, but appreciable amounts of barium entered the duodenum within an hour. There were no comments concerning the pyloric antra or pyloric canals. In the third infant in whom no tumour was found at operation (Appendix: Baby 18, Series A) gastric distension was gross and the barium did not begin to leave the stomach for $\mathbf{2 0}$ minutes, after which its passage was uninterrupted but markedly slow.

Infants Developing Symptoms of Hypertrophic Pyloric Stenosis after the Age of 2 Weeks. During the period January, 1947, to December, 1954, 23 babies were admitted to general hospitals in Leeds who were (i) born in Leeds Maternity Hospital, (ii) diagnosed at varying times after discharge from that hospital as suffering from hypertrophic pyloric stenosis and (iii) successfully operated on in a Leeds hospital. The routine clinical neonatal records kept during the time these 23 infants were under observation in the Maternity Hospital have been reviewed.

The ages of the 23 babies at the time of discharge from the Maternity Hospital ranged from 10 to 12 days. Histories taken at the time the babies were admitted to general hospitals for operation indicated that symptoms of pyloric stenosis first appeared in four infants after the age of 28 days; in five infants in the fourth week; and in 10 infants in the third week of life. Clinical records made in the Maternity Hospital of these 19 babies revealed that in every case the neonatal progress had been entirely uneventful.

In the remaining four babies the onset of symptoms of gastric obstruction occurred on the fourteenth day of life in two cases and on the thirteenth day in the other two cases. Two of these infants (both breast fed) showed unsatisfactory weight progress. In one of these cases there were no symptoms and in the other there was occasional vomiting which was neither copious nor forceful.

The four infants who developed signs of pyloric obstruction within a few days of discharge were born before the present investigation had begun and were not examined in the Maternity Hospital for the presence of a palpable tumour.

Pathological Examination of Pyloric Musculature of Newly Born Infants Dying from Whatever Cause. The pyloric musculature of 30 newly born babies born in the Leeds Maternity Hospital at differing periods of gestation and dying within 10 days of birth from a variety of causes was examined pathologically. The examinations were carried out by Dr. A. R. Macgregor, of the Royal Hospital for Sick Children, Edinburgh, and included microscopical studies. During the same period Dr. Macgregor carried out similar studies on babies who died in maternity hospitals in Edinburgh. This series consisted of 45 stillborn and 63 liveborn infants.

Macroscopically recognizable thickening in the region of the pylorus was noted in one baby and was shown microscopically to be due to the presence of clusters of heterotopic pancreatic cells. No abnormal macroscopical or microscopical features were found in the pyloric musculature of any of the remaining 137 infants.

\section{Discussion}

Comparing them with those encountered in older infants referred for operation on account of hypertrophic pyloric stenosis, the tumours (with six exceptions) palpated in the present investigation, lacked conclusiveness in one or other of their characteristics. In this respect the findings resemble in general terms those reported by Wood and Astley (1952) in cases described as 'Pseudo-pyloric Stenosis'. Whereas, however, the average age of onset of vomiting in their cases was 3 weeks, vomiting (or regurgitation) began in the first week of life in all the infants included in Series $A$ of the present study. While all tumours in the present study were defined, some lacked precise definition. 
All were firm in consistence, but the firmness conveyed a sense of hard solidity in only a small minority. The tumours tended to acquire increased definition and firmness as successive rhythmic contractions accompanied gradual filling of the stomach in the way characteristic of tumours in older infants with hypertrophic pyloric stenosis. Contraction and relaxation of the tumours in this investigation were, however, more deliberate and more sluggish. The sudden contraction and sudden relaxation suggestive of violent seizure and release commonly appreciated towards the end of a feed in the older infant with classical pyloric stenosis were present in only five cases in the present investigation. The tumour of pyloric stenosis, which becomes extremely hard with almost violent suddenness, often acquires a 'spinning top' shape with a notably sharp circumferential edge. In the present investigation these characteristics were met with in only five infants.

The Nature of the Tumour. What was the nature of the contractile tumour palpated in 25 symptomless cases (Series B) and in 17 cases (Series A) with unsatisfactory weight progress associated with recurrent posseting, regurgitation or vomiting? There were no fatal cases in either series and no material was available for pathological study. Three babies were referred for operative treatment which was successful in each instance. In two of these infants (Appendix: Baby 3, Series $A$ and Baby 9, Series A) doubts were felt concerning the exact significance of the tumours. In one the tumour seemed to be minute in size although subject to violent and intensely hard spasmodic contractions. In the other the tumour was hard, of small as distinct from minute size, and unusual in being ring-shaped. At operation, small but appreciable tumours were found in each of these two cases. In the third baby (Appendix: Baby 18, Series A) the findings were wholly different. A large tumour was palpated which resembled in every respect, i.e., hardness, shape, rhythm, contraction and spasm, those felt in well established cases of true pyloric stenosis in older infants. Radiological screening showed acute gastric retention. At operation there was no visible tumour but complete symptomatic relief followed a Rammstedt operation.

This last case is evidence that a large, hard contractile tumour can be felt in the absence of organic change in the musculature of the pylorus. Despite the absence of organic change vomiting was unmistakably forcible and contributed to a loss of $1 \frac{1}{2} \mathrm{lb}$. in weight by the eighth day of life. By way of contrast tumours which eventually disappeared spontaneously, were felt in 16 symptomless infants.
These cases would seem to provide further evidence that a palpable contractile pyloric tumour is not of itself proof of organic changes in the structure of the gastric musculature. Indeed, the finding of 25 tumours in a small control series of 115 healthy newborn infants suggests that the presence of a detectable tumour without associated symptoms is not uncommon.

If this view is accepted, the question arises, What is the significance of a tumour in such circumstances. If not evidence of organic muscular change is it evidence of disturbed gastric function? It is clearly impossible to draw any definite line of demarcation between normal and abnormal gastric motor function. The 25 infants in Series B in whom tumours were felt in the absence of symptoms cannot be said to have shown evidence of disturbed gastric function. This applies even to those few babies in whom peristalsis was seen and in whom the results of gastric lavage contained an unusual amount of mucus. On the other hand there are grounds for attaching greater significance to the tumours felt in infants included in Series A. In this series tumours were palpated in no fewer than 17 of the 21 babies and in each instance were associated with gastric symptoms, an unsatisfactory weight progress, pronounced peristalsis and excessive gastric secretion of mucus. Mucus in considerable amounts suggests gastric irritation. Visible pronounced peristalsis suggests intensified gastric motor activity and could be interpreted as a response either to local irritation or to an element of obstruction. If this explanation is correct, and if the suggestion (Cole Collaborators, 1932) that the circular muscular coat of the pyloric end of the stomach has a propulsive as well as a sphincteric function, frustrated peristaltic activity may well be followed by muscular spasm. Spasm of a segment of pyloric musculature would explain detection of a tumour, stimulated by filling of the stomach with a feed, into recurrent contraction and relaxation.

The presence of a detectable tumour cannot of itself be interpreted as evidence of disturbed gastric motor function. It is interesting, however, to note that among the infants who were screened, possibly abnormal motor function was a feature of 20 of the 25 infants with a palpable tumour and in only six of the 29 infants in whom no tumour was felt. Apparently disturbed gastric motor function was of two kinds: delay in the appearance of peristalsis and in emptying of the stomach, and notably vigorous peristaltic activity with extremely rapid passage of barium into the duodenum. In infants with a palpable tumour, vigorous motility was twice as common as delayed emptying of the stomach. 
These observations might be accounted for by spasm of the pyloric musculature which could explain also the absence at operation of a contractile pyloric tumour previously palpated during clinical examination.

The radiological findings are interesting in view of Henderson's (1942) opinion that gastric motility is much more variable in the newborn than in adults; and of Smith's (1951) statement that the stomach empties more slowly in the newborn period than at any other time of life. Discussing the passage of fluid from the stomach Smith attaches less significance to gastric peristaltic activity than to what he describes as 'unpredictable relaxation of the pylorus and massive, gentle non-peristaltic movement'.

Opinions differ concerning the existence of pylorospasm as a separate clinical entity, and it is not mentioned in recent editions of a number of textbooks. Nelson (1954) suggests that pylorospasm is mistakenly applied to those infants in whom frequent and often violent vomiting is to be ascribed to abnormal excitability and overactivity. These were not features of infants studied in this investigation. Parmelee (1954), on the other hand, gives simple pylorospasm as a condition to be differentiated from pyloric stenosis, but discussing diagnosis states specifically that a tumour is not found in cases of pylorospasm. This opinion agrees with that originally advanced by Thomson (1921) who mentioned that in pylorospasm vomiting sometimes 'recurs at short intervals until the stomach is emptied'-a feature in several of the infants with tumours in the present study.

Wood and Astley (1952) in their study of vomiting of uncertain origin in infants, describe 12 babies in whom they attributed vomiting to pylorospasm. A tumour differing in consistence from that of the tumours of pyloric stenosis was felt in eight of their cases. Five infants were operated upon and found to have no hypertrophy. Wood and Astley do not discuss the possible nature of the tumour felt in these babies, but Astley (1952) is satisfied that infantile pylorospasm is a cause of occasional difficulty in radiological diagnosis. The cases described by Wood and Astley resemble those which are the subject of the present article in the association of a tumour with vomiting attributed to pylorospasm; but they differ in that the babies were older, fretful and in most instances artificially fed.

Remembering that this article is concerned with infants of less than 2 weeks of age, there is at least a possibility that one or more of the pyloric tumours operated upon were in fact evidence not of muscular hypertrophy but of muscular spasm. Mention has already been made of the two babies in Series A in whom very small tumours were found at operation (Appendix: Babies 3 and 9, Series A). Clinical diagnosis in each of these cases was one of pyloric stenosis. Immediate and dramatic clinical improvement followed Rammstedt operations. This of itself is not conclusive evidence that the very small tumours consisted of hypertrophied muscle tissue. No less dramatic and no less permanent improvement followed incision of the pyloric sphincter in the case in which no tumour was found at operation (Appendix: Baby 18, Series A). Instances have been reported (Browne, 1931; Thompson and Gaisford, 1935) of comparable results following Rammstedt operations on older infants in similar circumstances. On the other hand vomiting did not cease suddenly in an infant reported by Field (1941) and in two babies in Wood and Astley's series on whom Rammstedt operations were performed.

The purpose of this article is not to discuss the aetiology of pyloric stenosis but to stimulate investigation into the incidence and significance of palpable contractile pyloric tumours in the newly born. Although the present investigation consists of no more than a pilot study it is considered that contractile pyloric tumours can be palpated with unexpected frequency in the newly born, and that there is justification for believing that these tumours are usually evidence of muscular spasm and not muscular hypertrophy. The incidence of tumours in Series A and B was high. Were the clinical detection of a tumour evidence of organic muscular changes, it would be reasonable to expect pathological evidence of changes in the pyloric musculature in a proportion of newborn babies dying from whatever cause in the first two weeks of life. No such evidence was forthcoming as a result of Dr. Macgregor's detailed studies.

The significance to be attached to palpable contractile pyloric tumours in the newly born is of importance from the point of view of diagnosis, management and aetiology. That true pyloric stenosis can occur in the early days of life is unquestionable. A limited number of cases have been reported in newly born infants. Nevertheless, the incidence of tumours in the present study is far in excess of the believed incidence of pyloric stenosis in the newborn or, for that matter, in infants of any age. It might be argued that the tumours reported in this article were examples of hypertrophied pyloric musculature undergoing the spontaneous resolution which is known to occur in a proportion of older infants. In the light of experience with older infants, however, it is highly improbable that 
any organic condition could be capable of such a high incidence of spontaneous resolution. Moreover, it is difficult to visualize resolution of an organic structural condition giving rise to the rapid clinical improvement characteristic of cases in Series A.

No reason can be advanced for the finding that a number of tumours in the present study were palpable for several weeks or months. Persistence was not accompanied by any symptoms. It is interesting to note in this connexion that Caffey (1945), discussing pyloric stenosis, states that radiological evidence of 'spasm of the antrum and stenosis of the pyloric channel may persist for years after surgical treatment and the complete relief of symptoms'.

Then, too, there is the possible significance of pylorospasm as a precursor or a contributory cause of pyloric hypertrophy. Findings in the present study do not suggest that a connexion exists between spasm and hypertrophy. Of infants with a tumour in the first two weeks of life none developed hypertrophic stenosis; and no tumour was palpated on examination on the eighth day of life in the only infant in the control series who later developed hypertrophic stenosis. Moreover, study of the notes of 23 infants operated on in general hospitals for pyloric stenosis revealed no connexion between progress during the first 12 days of life and the development of obstructive symptoms in later infancy.

Genetic Considerations. The significance of tumours encountered in the newborn is of importance also in relation to genetic studies. Presentday parents include many individuals who have recovered after operative treatment for hypertrophic pyloric stenosis in infancy. If, as has been suggested, heredity plays a part in the causation of pyloric stenosis (Carter and Savage, 1951) an increased incidence of the condition in infants can be expected (Brit. med. J., 1954). It is important that future genetic studies do not attach too much significance to retrospective records of tumours in the early days of life which, although detected clinically and suspected radiologically, were not confirmed at operation.

Diagnosis. Finally, there is the vital question of decision as to treatment. A diagnosis of hypertrophic pyloric stenosis in the first two weeks of life calls for early operation. Contrary to what is experienced in older infants the utmost difficulty may be encountered in arriving at a final diagnosis despite repeated examination and despite radiodiagnostic help. The present study permits of no more than very tentative conclusions. These may be summarized as follows:

(1) Recurrent vomiting, failure to gain weight, visible peristalsis and a palpable tumour may be present in cases of pylorospasm or pyloric stenosis.

(2) Excessive gastric secretion of mucus is suggestive of pylorospasm rather than pyloric stenosis.

(3) Vomiting due to stenosis is usually more severe than that arising from pylorospasm, but this is not invariable.

(4) Vomiting due to stenosis, although copious, is unlikely to be truly projectile; it tends rather to be deliberate, resembling the flow from the spout of a tilted watering can.

(5) After a feed vomiting due to pylorospasm may occur once, twice or thrice at short intervals, whereas one copious vomit serves to empty a stomach obstructed by true stenosis.

(6) The tumour due to hypertrophic stenosis is harder and contracts more fiercely than that found in cases of pylorospasm; otherwise there is no constant distinguishing feature between tumours encountered in the two conditions.

(7) Recognizing that allowance must be made for exceptions, it can be said that in general the infant with pylorospasm fails to progress over a period of a few days while the condition of the baby with pyloric stenosis deteriorates progressively but not necessarily with great rapidity.

(8) Peristalsis tends to be more marked in cases of true pyloric stenosis.

(9) Observations concerning the stools have no reliable significance in differential diagnosis.

(10) Radiography has only a limited value in assessing the significance of a pyloric tumour in the first 10 to 14 days of life.

(11) Symptoms due to pylorospasm settle within a few days with treatment in the form of repeated gastric lavage. The need to try antispasmodic drugs did not arise in the present investigation, but should be kept in mind.

As already emphasized these conclusions are tentative. They are intended to apply only to infants of less than 2 weeks of age and are published in the hope that they will stimulate organized study.

\section{Summary}

A pilot clinical study intended to investigate the significance of palpable contractile pyloric tumours in newborn babies of under 2 weeks of age is described.

Clinical material consisted of two series of cases, 21 infants in whom failure to progress was associated with recurrent vomiting or regurgitation, 
and 115 infants whose progress was satisfactory with no signs or symptoms of disturbed health.

Palpable contractile pyloric tumours were detected in $\mathbf{1 7}$ of the $\mathbf{2 1}$ infants in the first group and in $\mathbf{2 5}$ of the 115 infants in the second.

Details are given of the system of examination employed, the standards adhered to in determining the presence of a tumour, the methods of clinical management, and the results of $x$-ray screening.

The previously recorded neonatal histories of 23 infants who, after discharge from a maternity hospital, were subsequently operated on in general hospitals for pyloric stenosis are reviewed.

The results of follow-up studies are described.

The view is held that a palpable contractile pyloric tumour is present in a proportion of newborn infants in whom there is no organic disease.

It is suggested that in the absence of muscular hypertrophy the tumour is evidence of segmental spasm of the pyloric musculature, which is not to be regarded as necessarily evidence of abnormal function.

Instances of spontaneously subsiding recurrent vomiting (and regurgitation) in infants in whom tumours were felt are described and the suggestion made that these cases are examples of pylorospasm of a degree indicative of abnormal function.

Difficulties in the way of differentiating pylorospasm from hypertrophic pyloric stenosis in the early days of life are outlined, and tentative suggestions advanced.

No rigid conclusions have been arrived at and the purpose of the study is to focus attention on a subject which is considered to merit organized research.

\section{APPENDIX}

\section{Summarized Clinical Observations on Certain Infants with Prominent Physical Characteristics and/or Severe Symptoms}

Baby 3, Series A. Male, born in hospital, first child, birth weight $6 \mathrm{lb} .15 \mathrm{oz}$. Artificial feeding throughout; regurgitation on third and fourth day; frequent 'welling' but not projectile vomiting, with mucus, from seventh day; flat weight curve from second day. Spasmodic local thickening of pylorus palpated on tenth and eleventh day; minute 'tumour' with sudden phases of intense hardness felt on twelfth and thirteenth day; Rammstedt's operation performed on fourteenth day-very small tumour; subsequent progress uninterrupted (Fig. 1).

Baby 9, Series A. Male, born at home, second child, birth weight $7 \mathrm{lb}$. $12 \mathrm{oz}$. Breast fed throughout; frequent vomiting began on tenth day; admitted to hospital on 13th day. Frequent projectile vomiting, occasionally blood stained, copious mucus; fourteenth and fifteenth day pylorus felt but no tumour detected; sixteenth day pylorus notably firm; seventeenth day a very narrow, ring-like tumour felt to contract and relax as if in intense spasm; flat weight curve from admission; eighteenth day Rammstedt's operation-small tumour; subsequent progress uninterrupted.

Baby 11, Series A. Male, born in hospital, birth weight $7 \mathrm{lb} .9 \mathrm{oz}$. Breast fed. Regurgitation began on second day, and persisted until eighth day; occasional vomiting; excess mucus; tumour palpated on fourth, fifth and sixth days-spheroidal, hard and large-very easily felt and clearly defined. Weight curve flat from second to eighth day. Treatment limited to gastric lavage. Clinical improvement began in the second week of life. The tumour decreased gradually in size but was detectable until 10 weeks of age.

Baby 18, Series A. Male, born at home, first child, birth weight $7 \mathrm{lb}$.

Noticed to be exceptionally 'mucousy' from birth, excess mucus giving rise to retching and coughing. Offered breast and later expressed breast milk; all feeds exceeding $1 \mathrm{oz}$. in amount were immediately vomited. Stools infrequent and small. Admitted to hospital on eighth day of life markedly dehydrated and with weight of $5 \frac{1}{2} \mathrm{lb}$. Results of gastric lavage contained a very large amount of mucus and undigested curds; feeding followed rapidly by gross distension of stomach and later by forcible 'spouting' vomiting. Pronounced peristalsis; readily palpable, surprisingly large, firm contractile tumour of 'spinning top' shape. $X$-ray screening showed enormous gastric distension and delay in start of emptying for over 20 minutes. Laparotomy on ninth day of life; no visible tumour but Rammstedt's operation performed. Thereafter uninterrupted progress. Tumour not palpated on re-examination at the age of 1 month (Fig. 4).

Baby 14, Series B. Male, born in hospital, first child, birth weight $7 \mathrm{lb}$. $1 \mathrm{oz}$. Uneventful clinical progress from time of birth to last examination at the age of 4 months. Feeding at the breast throughout.

On sixth day of life tumour palpated-size of large pea, rhythmically contractile with filling of stomach, notably firm to point of hardness; no vomiting but considerable mucus in results of gastric lavage. Slight peristalsis. Examined at fortnightly intervals, tumour lessened in size and definition, disappearing at the age of 3 months.

Baby 97, Series B. Male, born in hospital, first child, birth weight $7 \mathrm{lb} .10 \mathrm{oz}$. Uneventful clinical progress apart from slight nasal catarrh in the second week. Breast feeding throughout.

On seventh day of life readily felt contractile tumoursmall marble in size, spheroidal in shape, gradual contraction and relaxation, firm but not hard, minimal peristalsis. Examined at three weekly intervals, tumour disappeared gradually within six weeks.

I am indebted to the senior obstetrical staff of the Leeds Maternity Hospital for allowing me access to the babies of mothers under their care; to mothers of babies included in the control series for permission to examine 
their babies; to Dr. A. R. Macgregor for pathological assistance and advice; and to Professor A. S. Johnstone and Dr. J. M. Winn for radiological help and reports. The enquiry would not have been possible without the enthusiastic help received from the nursing staff of Blackburn Ward, the Maternity Hospital, and I am especially grateful to the Departmental Sister, Miss M. Pattullo, and to Sisters M. J. Neasham and N. Sixsmith. Mrs. G. M. Turner (radiographer) gave great help in the making of arrangements for $x$-ray screening. I wish to acknowledge also help received in the course of discussion with Professor A. Durward and Dr. W. Goldie; and the reports of operative findings given me by Mr. H. S. Shucksmith. Dr. E. C. Allibone kindly lent records of infants admitted under his care in the General Infirmary: and Mr. L. Jolly, Librarian, The Royal College of Physicians, Edinburgh, gave me valuable assistance in providing references. I am greatly indebted to Miss J. Akester, Superintendent of Health Visitors, the City of Leeds Maternity and Child Welfare
Department, and her colleagues for invaluable help in following up many of the babies.

Astley, R. (1952) Brit, $J$ RERENCES

Astley, R. (1952). Brit. J. Radiol., 25, 342.

Brit. med. J. (1954). Leader, vol. 2, p. 583.

Browne, D. (1931). Archives of Disease in Childhood, 6, 129.

Caffey, J. (1945). Pediatric X-Ray Diagnosis, p. 428. Chicago.

Carter, C. O. and Savage, T. R. (1951). Archives of Disease in Childhood, 26, 50.

Cole Collaborators (1932), Radiology, 18, 886.

Fole Collaborators (1932). Radiology, 18, 886.

Field, C. E. (1941). Proc. roy. Soc. Med., 35, 59.
Henderson, S. G. (1942). Amer. J. Roentgenol., 48, 302.

Nelson, W. E. (1954). Textbook of Pediatrics, 6th ed., p. 683. Philadelphia.

Parmelee, A. H. (1954). In Brennemann's Practice of Pediatrics, vol. 3, ch. 5. Hagerstown, Maryland.

Smith, C. A. (1951). The Physiology of the Newborn Infant, 2nd ed., p. 184. Oxford.

Thomson, J. (1921). The Clinical Study and Treatment of Sick Children, 3rd ed., p. 291. Edinburgh.

Thompson, W. A. and Gaisford, W. F. (1935). Brit. med. J., 2, 1037. Wood, B. S. B. and Astley, R. (1952). Archives of Disease in Childhood, $27,562$. 